\title{
BMJ Open Moxibustion for the treatment of pressure ulcers: study protocol for a pilot, multicentre, randomised controlled trial
}

\author{
Qin-hong Zhang, ${ }^{1}$ Jin-huan Yue ${ }^{2}$ Chao-ran Li, ${ }^{1}$ Zhong-ren Sun ${ }^{1,2}$
}

To cite: Zhang Q-hong, Yue J-huan, Li C-ran, et al. Moxibustion for the treatment of pressure ulcers: study protocol for a pilot, multicentre, randomised controlled trial. BMJ Open 2014:4:e06423.

doi:10.1136/bmjopen-2014006423

- Prepublication history for this paper is available online. To view these files please visit the journal online (http://dx.doi.org/10.1136/ bmjopen-2014-006423).

J-hY and Q-hZ contributed equally.

Received 20 August 2014 Revised 1 September 2014 Accepted 8 September 2014

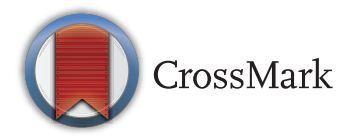

${ }^{1}$ Department of Acupuncture and Moxibustion, Second Affiliated Hospital of Heilongjiang University of Chinese Medicine, Harbin, People's Republic of China 2Department of Acupuncture and Moxibustion, First Affiliated Hospital of Heilongjiang University of Chinese Medicine, Harbin, People's Republic of China

Correspondence to Professor Zhong-ren Sun; sunzhongren2011@163.com

\section{ABSTRACT}

Introduction: Pressure ulcers are common in the elderly and immobile. Currently, there are few proven effective treatments for pressure ulcers. This trial aims to evaluate the feasibility, efficacy and safety of moxibustion for pressure ulcers.

Methods/analysis: This is a multicentre, two-armed, parallel-design randomised controlled trial (RCT). 30 eligible patients with pressure ulcers will be randomised in a ratio of 1:1 to the treatment group and control group. The participants in the treatment group will undergo indirect moxibustion for 30 min before application of a dressing, one session daily, five sessions weekly for 4 weeks. The patients in the control group will only receive a dressing, applied in the same way as in the treatment group. Both groups will be followed up for 3 months. The primary outcome measures will be wound surface area (WSA) and proportion of ulcers healed within trial period (PUHTP). The secondary outcomes will be the Pressure Ulcer Scale for Healing (PUSH Tool), visual analogue scale (VAS) and adverse events. All outcomes will be evaluated at the beginning of the study, at the end of the second week, at 4 weeks after randomisation and at 1 and 3 months after treatment cessation.

Ethics/dissemination: This trial has undergone ethical scrutiny and been approved by the ethics review boards of First Affiliated Hospital of Heilongjiang University of Chinese Medicine and Second Affiliated Hospital of Heilongjiang University of Chinese Medicine (Permission number: HZYEYLP2014). The results of this study will provide clinical evidence for the feasibility, efficacy and safety of moxibustion for pressure ulcers.

Trial registration number: ChiCTR-TRC-13003959.

\section{BACKGROUND}

A pressure ulcer (ie, bedsore, pressure sore, decubitus ulcer) is defined as a localised injury to the skin and underlying tissue layers affecting the muscle, tendon and bone as a result of constant pressure due to impaired mobility. ${ }^{1}{ }^{2}$ Pressure ulcers are often caused by pressure, shear, friction or a combination of these. ${ }^{3}$
Common sites for pressure ulcers include the back of the head, shoulder blade, elbow, lower back, hip, pelvic bone, ankle and heel. ${ }^{4}$ Pressure ulcers are generally classified into four stages (stages I, II, III and IV) according to the European Pressure Ulcer Advisory Panel and National Pressure Ulcer Advisory Panel guidelines (EPUAP/NPUAP). ${ }^{3}$

The prevalence of pressure ulcers across studies varies from $8.8 \%$ to $53.2 \%,{ }^{5-8}$ and incidence rates range from $7 \%$ to $71.6 \%{ }^{7-10}$ It has been estimated that the annual treatment cost of pressure ulcers ranges from 1.4 to 2.1 billion GBP (Great British pound), which is broadly equal to the total UK National Health Service expenditure on mental illness, or the total cost of community health services. ${ }^{11}$ Effective and adequate prevention is an important issue for patients, clinicians and policymakers.

Moxibustion is a popular and safe intervention used to manage various conditions, such as breech presentation, ${ }^{12}$ facial paralysis, ${ }^{13}$ chronic fatigue, ${ }^{14}$ knee osteoarthritis ${ }^{15}$ and type 2 diabetes mellitus. ${ }^{16}$ In addition, moxibustion has been found to be effective in treating pressure ulcers. ${ }^{17}{ }^{18}$ However, most prior case reports, case series or clinical trials were without a control group, resulting in poor quality of these studies. Considering these methodological flaws, we will conduct a multicentre RCT to investigate the effectiveness and safety of moxibustion. The results of this study will provide evidence for the feasibility of a large clinical trial, and yield data to determine the appropriate sample size for future large-scale RCTs of moxibustion in patients with pressure ulcers.

\section{METHODS/DESIGN \\ Objective}

The primary objective of this study is to assess the feasibility of moxibustion treatment for pressure ulcers and obtain preliminary 
safety and efficacy data to inform a future, larger RCT in the same patient population.

\section{Design}

This is an assessor-blinded and analyst-blinded, randomised, controlled clinical trial with two parallel arms. The trial will be conducted at two clinical centres in China: First Affiliated Hospital of Heilongjiang University of Chinese Medicine and Second Affiliated Hospital of Heilongjiang University of Chinese Medicine, between 29 May 2014 and 31 December 2016. This protocol has been approved by the ethics review boards of First Affiliated Hospital of Heilongjiang University of Chinese Medicine and Second Affiliated Hospital of Heilongjiang University of Chinese Medicine with permission number HZYEYLP2014. Written informed consent will be obtained from all study participants prior to enrolment. JHY will approach potential participants and invite them to participate in the study, and CRL will ask them to complete the consent process.

Eligible patients will be randomised in a ratio of $1: 1$ to the treatment group (moxibustion plus dressing) and control group (dressing), and receive treatment for 4 weeks. Patients will be followed up for 3 months (figure 1 and table 1). Outcome measures will be assessed at baseline, as well as at the end of the second and fourth week after randomisation, and at 1 and 3 months after treatment cessation. Results will be analysed by professionals blinded to the group allocation. This protocol has been registered with the Chinese Clinical Trials Register, a registry in the WHO Registry Network.

\section{ELIGIBILITY}

\section{Inclusion criteria}

Participants will be included if they fulfil the following criteria: (1) aged 18-75 years; (2) pressure ulcers belonging to stage II or III according to the EPUAP/ NPUAP guidelines ${ }^{3}$ and (3) at least one pressure ulcer.

\section{Exclusion criteria}

Patients with any of the following conditions will be excluded because of safety concerns and possible sensation loss interfering with evaluation of pain intensity: (1) undergoing other therapies that might interfere with the ability to heal, such as corticosteroid therapy, radiation therapy or chemotherapy for cancer; (2) infected pressure ulcers; (3) complications of diabetes mellitus and (4) severe diseases, such as liver, cardiac and kidney diseases, and relevant serious complications.

\section{Randomisation and allocation concealment}

In this trial, randomisation will be performed at the Good Clinical Practice (GCP) Center of Second Affiliated Hospital of Heilongjiang University of Chinese Medicine. Thirty participants who meet the eligibility criteria will be assigned in a ratio of $1: 1$ to the treatment group and control group. Patients will be randomised using a computerised number generator through the stratified block randomisation method of the Statistical Analysis System (SAS) package (V.9.1.3; SAS Institute Inc, Cary, North Carolina, USA) by a statistician with no clinical involvement in this trial. The allocation will be concealed in sequentially numbered, opaque, sealed envelopes containing the randomisation assignments. Allocation concealment will be
Figure 1 Flow chart of study process.

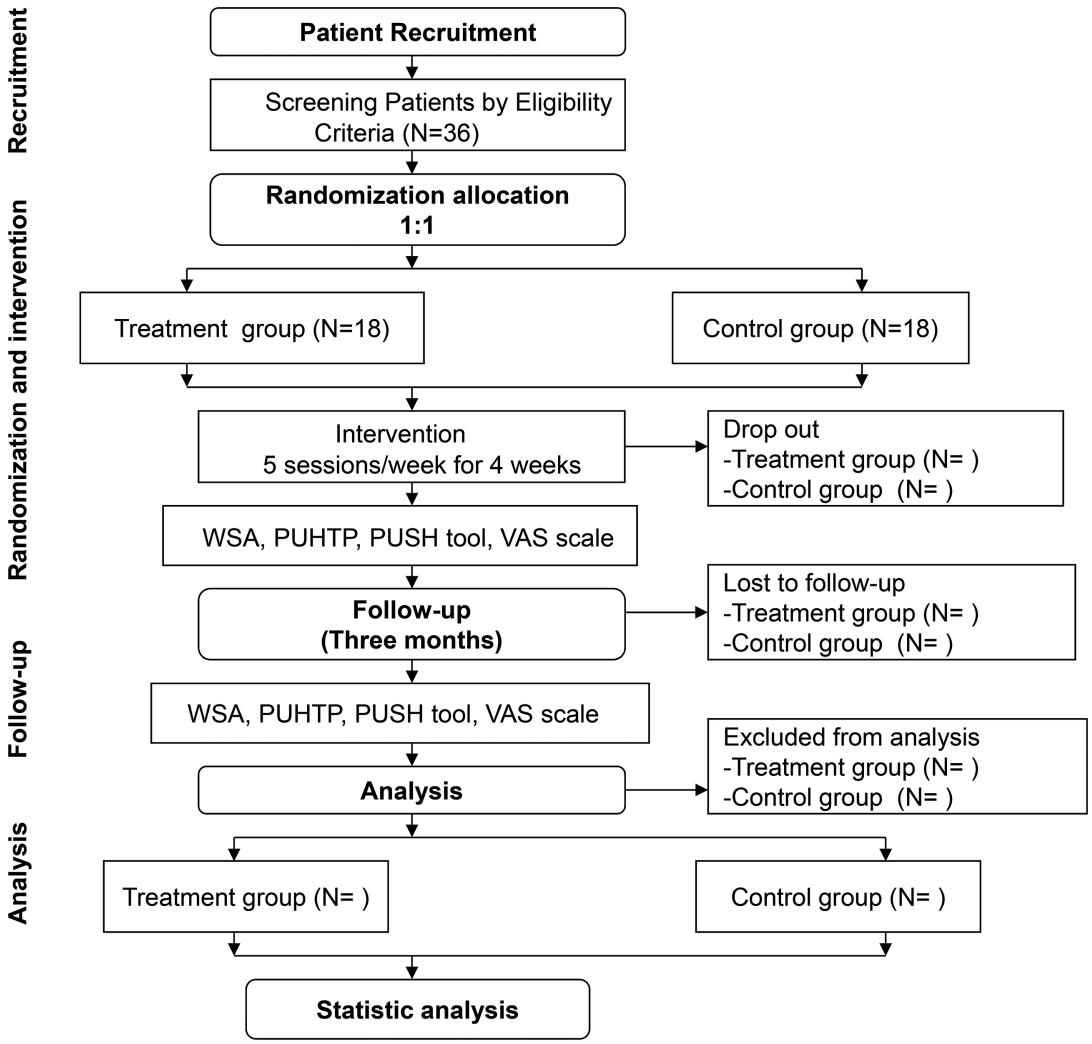


Table 1 Time of visits and data collection

\begin{tabular}{|c|c|c|c|c|c|c|}
\hline & \multirow{2}{*}{$\begin{array}{l}\text { Baseline } \\
-1 \text { Week }\end{array}$} & \multicolumn{3}{|c|}{ Treatment phase } & \multicolumn{2}{|c|}{$\begin{array}{l}\text { Follow-up phase } \\
\text { (end of treatment) }\end{array}$} \\
\hline & & o Week & 2 Weeks & 4 Weeks & 1 Month & 3 Months \\
\hline \multicolumn{7}{|l|}{ Patients } \\
\hline Informed consent & $x$ & & & & & \\
\hline Sign the informed consent & & $x$ & & & & \\
\hline Medical history & $x$ & & & & & \\
\hline Physical examination & $x$ & & & & & \\
\hline Randomisation & & $x$ & & & & \\
\hline \multicolumn{7}{|l|}{ Intervention } \\
\hline Treatment group $(n=18)$ & & \multicolumn{5}{|c|}{$\begin{array}{l}20 \text { sessions of moxibustion plus } \\
\text { dressing }\end{array}$} \\
\hline \multicolumn{7}{|l|}{ Comparison } \\
\hline Control group $(n=18)$ & & \multicolumn{3}{|c|}{20 sessions of dressing } & & \\
\hline \multicolumn{7}{|l|}{ Outcomes } \\
\hline WSA & & $x$ & $x$ & $x$ & $x$ & $x$ \\
\hline PUHTP & & $x$ & $x$ & $x$ & $x$ & $x$ \\
\hline PUSH Tool & & $x$ & $x$ & $x$ & $x$ & $x$ \\
\hline VAS & & $x$ & $x$ & $x$ & $\times$ & $x$ \\
\hline \multicolumn{7}{|l|}{ Participant safety } \\
\hline Adverse events & & $x$ & $x$ & $x$ & $x$ & $x$ \\
\hline
\end{tabular}

broken only after the participant has met all selection criteria and completed the baseline assessments. The participants will know the allocated group, but the outcome assessors and data analysts will be masked to the intervention allocation. ${ }^{19}$

\section{Blinding}

In moxibustion research, it is not feasible to conceal allocation from the practitioners. In addition, it is not possible to prevent participants from knowing if they have received moxibustion treatment or dressing intervention, or both. The treatment and assessment will be performed independently. The outcome assessors and the data statistical analysts will be blinded to treatment allocation throughout the study.

\section{INTERVENTION}

\section{Treatment group (moxibustion plus dressing)}

The participants receiving moxibustion treatment will be treated indirectly at a distance of $2-3 \mathrm{~cm}$ from the wound surface for $30 \mathrm{~min}$ per session, with one session daily and five sessions weekly for 4 weeks. In addition, a dressing will be applied in one session daily and five sessions weekly for 4 weeks.

\section{Control group (dressing)}

The format of the dressing intervention will be the same as in the treatment group.

\section{OUTCOME MEASURES}

The wound surface area (WSA), proportion of ulcers healed within trial period (PUHTP), Pressure Ulcer
Scale for Healing (PUSH Tool) and visual analogue scale (VAS) will be the outcomes used to assess efficacy. Adverse events will be recorded to assess safety.

\section{Primary outcomes}

Wound surface area

The $\mathrm{WSA}^{20}$ of the pressure ulcers will be measured by the use of acetate tracing and subsequent planimetric determination. Ulcer tracings will be accomplished by outlining the pressure ulcer circumference onto a transparent film applied directly over the wound. Each ulcer will be traced three times by two assessors, respectively, in order to improve the accuracy of these tracings. A third assessor will determine the ulcer surface area from the wound tracing using a planimeter (KP-21C). All three assessors will be blinded to the identities of the patients and to the treatment group assignments.

\section{Proportion of ulcers healed within trial period}

Completely healed ulcers will be defined as $100 \%$ epithelisation or skin closure without drainage. ${ }^{20}$

\section{Secondary outcomes}

\section{Pressure ulcer scale for healing}

The PUSH Tool ${ }^{21}$ was developed by the NPUAP as a quick, reliable tool to monitor the change in pressure ulcer status. This tool categorises ulcers with respect to surface area, exudate and type of wound tissue. Subscores for each of these ulcer characteristics will be recorded and then added to obtain the total score. A comparison of total scores measured over time provides an indication of the improvement or deterioration in pressure ulcer healing. 
Visual analogue scale

The pain intensity of pressure ulcers will be assessed using the $100 \mathrm{~mm}$ VAS (0, absence of pain; 100, the worst pain imaginable). ${ }^{22}{ }^{23}$ In order to evaluate the clinical severity and impact on activities of daily life in patients with bedsores, the VAS is selected as a secondary outcome measurement.

\section{STATISTICAL METHODS}

\section{Sample size}

Although several studies have investigated the effects of moxibustion on pressure ulcers, no RCTs have assessed the effects of moxibustion on pressure ulcers. There is no previous study on which to base the sample size calculation. Therefore, this pilot study will evaluate the efficacy and safety of moxibustion and feasibility of clinical trials. Taking into account the minimum number of subjects necessary to evaluate the efficacy of moxibustion, this pilot study will include 36 participants (18 in each group) with an expected dropout rate of $20 \% .^{24}$

\section{Statistical analysis}

Data will be analysed by a statistician blinded to the group allocations using the Statistical Package for the Social Sciences (SPSS) V.17.0 statistical software package. Significant levels will be reported at $p<0.05$. Data analysis of baseline characteristics, and of primary and secondary outcomes will be based on the intention-to-treat (ITT) principle. If an adjustment for possible baseline incomparability is needed, analysis of covariance will be conducted. If the measurement data of WSA, PUSH and VAS have non-normal distributions, the Wilcoxon rank sum test will be used. If the measurement data have normal distributions, the $\mathrm{t}$ test will be used. In the case of proportions, the $\chi^{2}$ or Fisher exact test will be applied (eg, PUHTP).

\section{Patient safety}

Any adverse events (described as unfavourable or unintended signs, symptoms or diseases occurring after treatment) related to moxibustion treatment will be observed and reported by patients and practitioners during each patient visit. In addition, all vital signs and adverse events will be measured and recorded at each visit.

\section{Quality control}

All staff will be required to undergo special training before participating in the trial. For example, staff will be trained to select participants and to conduct the moxibustion intervention. The monitors will check study protocol compliance and informed consent documents and assess the progress of the study, including participant recruitment, moxibustion intervention and data quality, at each centre once a month. Dropouts and withdrawals from the study will be recorded through the intervention and follow-up periods.

\section{DISCUSSION}

The objective of this study is to evaluate the effectiveness and safety of moxibustion for treating pressure ulcers. We designed this study to determine the efficacy and safety of moxibustion in the treatment group (moxibustion plus dressing) versus the control group (dressing). The results of this study will determine if moxibustion is an effective therapy for pressure ulcers.

The outcomes of WSA, PUHTP, PUSH Tool and VAS are well-evaluated pressure ulcers. This pilot study will provide data on the efficacy of moxibustion for pressure ulcers through a follow-up period of 3 months after the completion of the treatment. An assessment of these outcomes will also be needed in a future long-term clinical trial.

In addition to appropriate outcome measures, the use of an appropriate control group is a critical issue in designing a high-quality clinical trial. The purpose of this study is to clarify whether moxibustion is effective in patients with pressure ulcers, and we have planned a pragmatic design using a dressing intervention in the control group. This pragmatic trial comparing moxibustion plus dressing with dressing alone can provide evidence focused on the effectiveness of moxibustion.

In conclusion, this pilot, assessor-blinded and analystblinded, multicentre RCT will investigate the efficacy and safety of moxibustion for pressure ulcers, assess the feasibility and relevance of a moxibustion therapy study design, and provide a clinical foundation for future, large-scale, multicentre clinical trials.

Acknowledgements This work was partly supported by the National Foundation of Natural Science of China (grant no. 81273823, 81303045, 81473761), Doctoral Fund of Ministry of Education of China (grant no. 20122327110007), the Key Project of Heilongjiang Ministry of Education (grant no. 12521z023), Foundation of Outstanding Innovative Talents Support Plan of Heilongjiang University of Chinese Medicine (grant no. 2012RCL01; 2012RCQ64).

Contributors J-hY and Q-hZ contributed equally to this work. Q-hZ and Z-rS conceived the study and designed the study protocol. J-hY and C-rL drafted the manuscript. Q-hZ and Z-rS sought funding and ethical approval. All authors contributed to the further writing of the manuscript as well as read and approved the final manuscript.

Competing interests None.

Patient consent Obtained.

Ethics approval The ethics review boards of First Affiliated Hospital of Heilongjiang University of Chinese Medicine and Second Affiliated Hospital of Heilongjiang University of Chinese Medicine.

Provenance and peer review Not commissioned; externally peer reviewed.

Open Access This is an Open Access article distributed in accordance with the Creative Commons Attribution Non Commercial (CC BY-NC 4.0) license, which permits others to distribute, remix, adapt, build upon this work noncommercially, and license their derivative works on different terms, provided the original work is properly cited and the use is non-commercial. See: http:// creativecommons.org/licenses/by-nc/4.0/

\section{REFERENCES}

1. Reddy M, Gill SS, Kalkar SR, et al. Treatment of pressure ulcers: a systematic review. JAMA 2008;300:2647-62. 
2. Whitney J, Phillips L, Aslam R, et al. Guidelines for the treatment of pressure ulcers. Wound Repair Regen 2006;14:663-79.

3. European Pressure Ulcer Advisory Panel and National Pressure Ulcer Advisory Panel. Prevention and treatment of pressure ulcers: quick reference guide. Washington DC: National Pressure Ulcer Advisory Panel, 2009.

4. Torpy JM, Lynm C, Glass RM. Pressure ulcers. JAMA 2003;289:254.

5. Davis CM, Caseby NG. Prevalence and incidence studies of pressure ulcers in two long-term care facilities in Canada. Ostomy Wound Manage 2001;47:28-34.

6. Tannen A, Bours G, Halfens R, et al. A comparison of pressure ulcer prevalence rates in nursing homes in the Netherlands and Germany, adjusted for population characteristics. Res Nurs Health 2006;29:588-96.

7. Zhang Q, Sun Z, Yue J. Massage therapy for preventing pressure ulcers. Cochrane Database Syst Rev 2013;(5):CD010518.

8. Zhang QH, Sun ZR, Yue JH, et al. Traditional Chinese medicine for pressure ulcer: a meta-analysis. Int Wound J 2013;10:221-31.

9. Scott JR, Gibran NS, Engrav LH, et al. Incidence and characteristics of hospitalized patients with pressure ulcers: state of Washington, 1987 to 2000. Plast Reconstr Surg 2006;117:630-4.

10. Whittington KT, Briones R. National prevalence and incidence study: 6-year sequential acute care data. Adv Skin Wound Care 2004;17:490-4.

11. Bennett G, Dealey C, Posnett J. The cost of pressure ulcers in the UK. Age Ageing 2004;33:230-5.

12. Zhang QH, Yue JH, Liu M, et al. Moxibustion for the correction of nonvertex presentation: a systematic review and meta-analysis of randomized controlled trials. Evid Based Complement Alternat Med 2013;2013:241027. http://dx.doi.org/10.1155/2013/241027
13. Wang HW, Wen X, Wei QL. Moxibustion at Baihui (GV 20) for intractable facial paralysis and its impacts on immunoglobulin. Zhongguo Zhen Jiu 2013;33:306-8.

14. Kim HG, Yoo SR, Park HJ, et al. Indirect moxibustion (CV4 and CV8) ameliorates chronic fatigue: a randomized, double-blind, controlled study. J Altern Complement Med 2013;19:134-40.

15. Xie HW, Chen RX, Xu FM, et al. Comparative study of heat-sensitive moxibustion in treatment of knee osteoarthritis. Zhongguo Zhen Jiu 2012;32:229-32.

16. Kim TH, Choi TY, Shin BC, et al. Moxibustion for managing type 2 diabetes mellitus: a systematic review. Chin J Integr Med 2011;17:575-9.

17. Kang LR, Ba HY. Moxibustion on the selected points according to meridians for pressure ulcers of 15 cases. J Clin Acupunct Moxibustion 2004;20:22.

18. Shi XJ. Moxibustion for pressure ulcers of 12 cases. J Clin Acupunct Moxibustion 1988;1:8.

19. Altman DG, Dore CJ. Randomisation and baseline comparisons in clinical trials. Lancet 1990;335:149-53.

20. Zhang QH, Yue JH, Sun ZR. Electroacupuncture for pressure ulcer: a study protocol for a randomized controlled pilot trial. Trials 2014;15:7

21. National Pressure Ulcer Advisory Panel (NPUAP). PUSH Tool. Washington DC: National Pressure Ulcer Advisory Panel, 2010.

22. Revill SI, Robinson JO, Rosen M, et al. The reliability of a linear analogue for evaluating pain. Anaesthesia 1976;31:1191-8.

23. Carlsson AM. Assessment of chronic pain. 1. Aspects of the reliability and validity of the visual analogue scale. Pain 1983;16:87-101.

24. Johanson GA, Brooks GP. Initial scale development: sample size for pilot studies. Educ Psychol Meas 2010;70:394-400. 